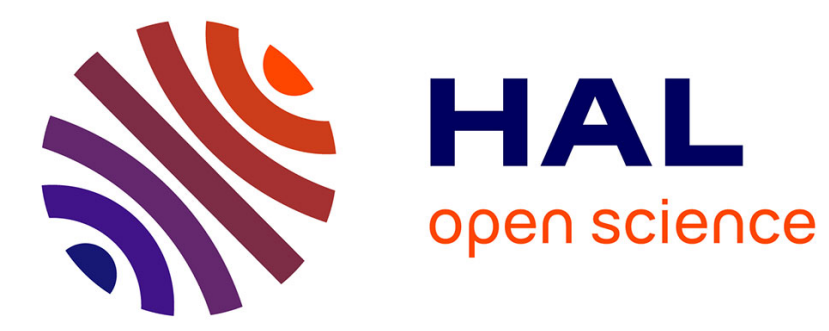

\title{
Integrated monitoring system for fall detection in elderly
} Shadi Khawandi, Bassam Daya, Pierre Chauvet

\section{To cite this version:}

Shadi Khawandi, Bassam Daya, Pierre Chauvet. Integrated monitoring system for fall detection in elderly. International Conference on Audio, Language and Image Processing (ICALIP 2012), 2012, Shanghai, China, Chine. pp.62-67, 10.1109/ICALIP.2012.6376585 . hal-03430621

\section{HAL Id: hal-03430621 \\ https://univ-angers.hal.science/hal-03430621}

Submitted on 16 Nov 2021

HAL is a multi-disciplinary open access archive for the deposit and dissemination of scientific research documents, whether they are published or not. The documents may come from teaching and research institutions in France or abroad, or from public or private research centers.
L'archive ouverte pluridisciplinaire $\mathbf{H A L}$, est destinée au dépôt et à la diffusion de documents scientifiques de niveau recherche, publiés ou non, émanant des établissements d'enseignement et de recherche français ou étrangers, des laboratoires publics ou privés. 


\title{
Integrated Monitoring System for Fall Detection in Elderly
}

\author{
${ }^{1}$ S. KHAWANDI, ${ }^{2}$ B. DAYA, ${ }^{3}$ P. CHAUVET \\ ${ }^{1}$ Phd student, University of Angers, Angers 49000-France \\ ${ }^{2}$ Prof., Lebanese University, 813-Lebanon \\ ${ }^{3}$ Prof., University of Angers, Angers 49000-France \\ chadi.khawandi@etud.univ-angers.fr
}

\begin{abstract}
Falling and its resulting injuries are an important public health problem for older adults. The National Safety Council estimates that persons over the age of 65 have the highest mortality rate (death rate) from injuries. The risk of falling increases with age; one of three adults 65 or older falls every year. Demographic predictions of population aged 65 and over suggest the need for telemedicine applications in the eldercare domain. This paper presents an integrated monitoring system for the detection of people falls in home environment. The system consist of combining low level features extracted from a video and heart rate tracking in order to classify the fall event. The extracted data will be processed by a neural network for classifying the events in two classes: fall and not fall. Reliable recognition rate of experimental results underlines satisfactory performance of our system
\end{abstract}

\section{Introduction}

With the population growing older and increasing number of people living alone, supportive home environments able to automatically monitor human activities are likely to widespread due to their promising ability of helping elderly people. Due to aging population, chronic diseases and their management costs are also on the rise. As medical science advances, people can live with better health and alone up to a very advanced age. Therefore, to let elderly people live in their own homes leading their normal life, while, and at the same time taking care of them requires new kinds of systems.
Many devices have been developed in the last few years for the falls detection [1][2], such as a social alarm, which is a wrist watch with a button that is activated by the person in case he/she suffers a fall, and wearable fall detectors, which are based on combinations of accelerometers and tilt sensors. However, these devices may present serious problems. The main problem with social alarms is that the button is often unreachable after a fall, especially when the person is panicked, confused, or unconscious. For the wearable sensors, these autonomous sensors are usually attached under the armpit, around the wrist, behind the ear's lobe or at the waist. However the problem of such detectors is that older people often forget to wear them [3][4], indeed their efficiency relies on the person's ability and willingness to wear them.

Also, recently some research has been done to detect falls based on using image processing approach. Some methods [7], [8] are based on analyzing aspect ratio of the moving object's bounding box; other methods [9], [13] used the normalized vertical and horizontal projection of segmented object as feature vectors. Some other approaches [6] detects falls using motion history image and human shape variation captured by though wall-mounted cameras to cover large areas. Other systems used the audio information or using 3D trajectory and speed of head to infer events [12]. These mechanisms tend to be more complex and need more additional cost. Despite the achievements that has accomplished in the recent years, there are still a main clear challenge to overcome:

With Visual fall detection; what appears to be a fall might not be a fall. Most of existing systems [8], [9], [10], [11] are unable to distinguish between real fall incident and an event when the person is lying or sitting down abruptly. 
The majority of injury-related hospitalizations for elderly are results of falls [5] and the situation will be much worse if the person cannot call for help. To overcome these problems, we present an integrated monitoring system for in-home people fall detection purpose within Ambient Assisted Living (AAL) context, with a particular interest to the problem of fall detection. It has in fact been demonstrated that, the delivery of assistance after a fall may reduce the risk of hospitalization by over $25 \%$ and of major injury or death by over $80 \%$ [6]. Furthermore, in order to prevent false alarms or missed falls, the use of an integrated system of webcam video and heart rate sensor, followed by a data fusion processed by a neural network, is provided. The developed system, in case of emergency, communicates with care holders and relatives of the assisted person through an ADSL-based gateway.

\section{Proposed approach}

Information Technology combined with recent advances in networking, mobile communications, and wireless medical sensor technologies offers great potential to support healthcare professionals and to deliver remote healthcare services, hence providing the opportunities to improve efficiency and quality and better access to care at the point of need.

Since one of the major causes of injury and fear for older people is fall, technology should be exploited to automatically provide as fast as possible call for assistance when needed, minimizing of course false alarms to improve the efficiency of the system/service. This paper proposes an integrated fall detector system shown in Figure 1 as a combination between 2 different commercial devices: a webcam and a hear rate accelerometer. Data extracted from the 2 sub-systems will be processed by neural network (MLP) in order to detect the fall. Once the fall is detected, an emergency alert will be activated automatically and sent to care holders through an internet-based home gateway.

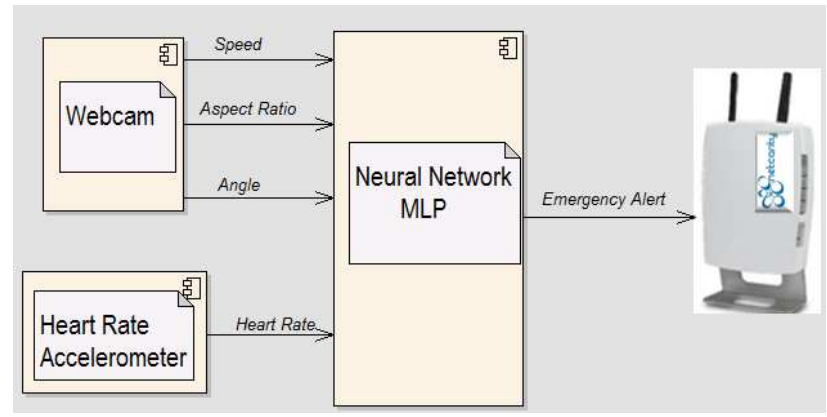

Figure 1. Overview of the integrated system

\subsection{Webcam monitoring system}

In this section, we present a vision-based analysis approach for monitoring human activities with a particular interest to the problem of fall detection. The proposed system is based on image processing in real time; this system detects the body and face of a person in a given area, collects data such as the aspect ratio, angle and speed of movement of the person, then send the extracted data to be processed by the MLP.

The system starts by removing the background. After the silhouette is acquired, the next step is the skin color detection which is an effective way often used to define a set of areas likely to contain a face or hands; then the system detects the face; for this, the shape of the detected object is compared with an ellipse; then features extraction is involved (speed of person's movement, distance between face and webcam, percentage of face present).

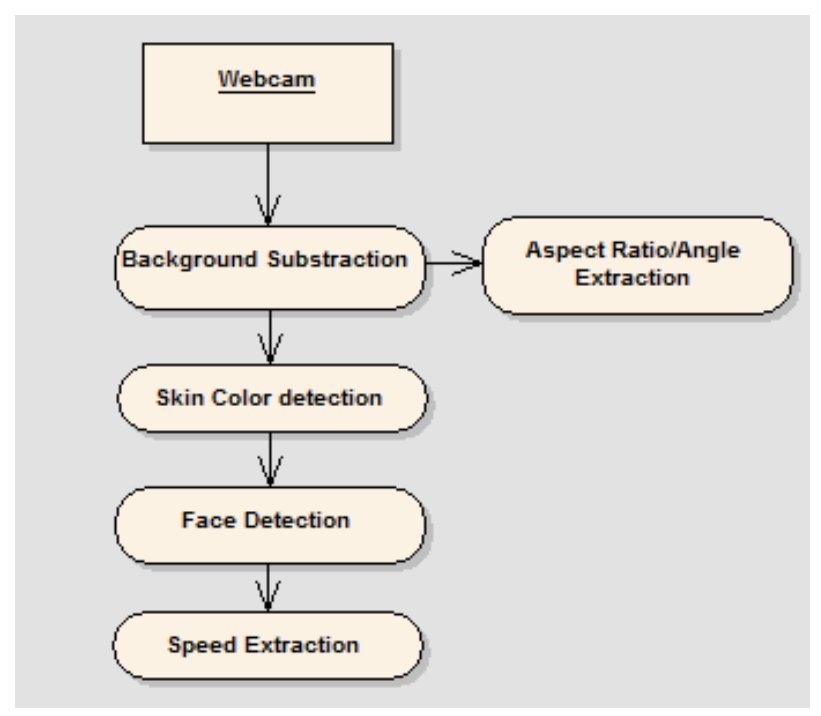

Figure 2. Overview of the webcam monitoring system

\subsubsection{Background Subtraction}

Background subtraction is a particularly popular method to detect moving regions in an image by differencing between current image and a reference background image in a pixel-by pixel way.

\subsubsection{Aspect Ratio and Angle Extraction}

- Aspect Ratio:

The aspect ratio of a person is simple yet effective features for differentiating normal standing pose from other abnormal pose (Figure 3). Aspect ratio of human body changes during fall. When a person falls, the 
height and width of his bounding box changes drastically (height/width). The range is from 0.15 to 6 (it can vary depending on the dimensions of the subject or on the scaling camera - to - image coefficients).

- Angle

Fall angle $(\theta)$ is the angle of a vertical line through the centroid of object with respect to the horizontal axis of the bounding box (Figure 3 ). Centroid (Cx, Cy) is the center of mass coordinates of an object. When a person is standing, we assume that he is in an upright position and the angle of a vertical line through the centroid with respect to horizontal axis of the bounding box should be approximately 90 degree. When a person is walking, the $\theta$ value varies from 45 degree to 90 degree. When a person is falling, the angle is always less than 45 degrees. For every frame we calculate the fall angle $(\theta)$ and if $\theta$ value is less than 45 degree, we confirm that the person is falling. The range is from 0 to 90 degrees.

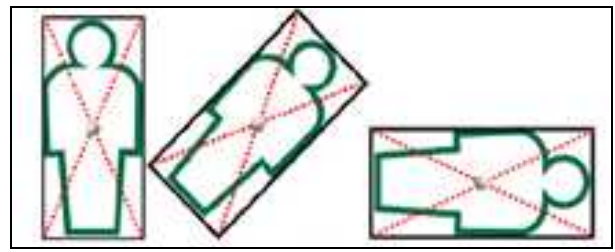

Figure 3. Bounding box and poses of human object

\subsubsection{Skin color and HSV detection}

The images captured by the webcams are then processed by the system to detect skin color. This is an effective technique for determining whether an image contains a face or hands. In this technique, the appropriate threshold values are defined for all the pixels in a color space. Different color spaces are used to represent skin color pixels: RGB, RGB standard, HSV (or HSI), YCrCb, and HSV. After the detection of skin color pixels, image filtering (erosion and dilation) is carried out.

\subsubsection{Face Detection- Approximated Ellipse}

After identifying the skin areas, it is necessary to distinguish the face. For this, the shape of the detected object is compared with an ellipse. This correlation technique is very effective and efficient.

Based on the comparison with an ellipse, we may have more than one image; such as the hand. In order to solve this issue, each image will be converted into binary image (black and white) then the white contour will be replaced by black. In this state, the object representing the hand goes black but the object representing the face becomes black except the eyes and mouth. After this transformation, we compute the white surface in each picture and the object having the greater white surface is the one of the face and in this case it is detected.

After calculating the white surface in each image, we found that the white surface in the face is greater than that in hand, that's why this intelligent system detects the face.

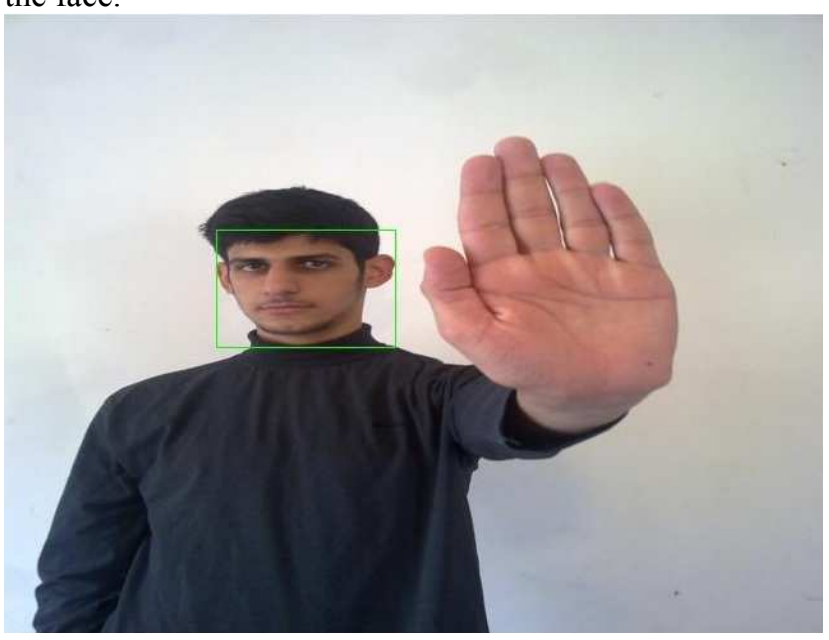

Figure 4. Face detected

\subsubsection{Speed Extraction}

One major point in recognition system is the feature extraction, i.e., the transition from the initial data space to a feature space that will make the recognition problem more tractable. So we analyze the shape changes of the detected face in the video sequence. The planar speed of movement is calculated using the following formula:

Planar speed $=$ distance $/$ time $($ pixel $/ \mathrm{s})$;

- Distance: between the same face in consecutive frames (pixel);

- Time: processing time between two consecutive frames.

The range is from 90 to 700 pixels (it can vary depending on the quality of the pictures).

\subsection{Wireless Heart Rate Monitor}

A heart rate monitor is a personal monitoring device which allows a subject to measure his or her heart rate in real time or record his or her heart rate for later 
study. Early models consisted of a monitoring box with a set of electrode leads which attached to the chest.

Modern heart rate monitors usually comprise two elements: a chest strap transmitter and a wrist receiver or mobile phone (which usually doubles as a watch or phone).

Newer versions include a microprocessor which is continuously monitoring the EKG and calculating the heart rate, and other parameters. These may include accelerometers which can detect speed and distance eliminating the need for foot worn devices.

Heart rate monitoring system with wireless transmission using zigbee is described in [14]. The system includes a bandage size heart beat sensing unit, a wireless communication link, and a networkable computer and a data base. [15] and [16], gives idea about an electronic stethoscope based on embedded processor and Bluetooth transmission which fulfill the shortages from auscultation.

This paper doesn't include the design of a heart rate monitor but we will use an existing heart rate monitor. Wifi heart rate belt (HRM-2823) could be connected on computer or Wifi operator mobile; this monitor has professional software providing online data exchange model that allow the heart rate belt keeps connecting with the PC and transmits data to PC in real time.

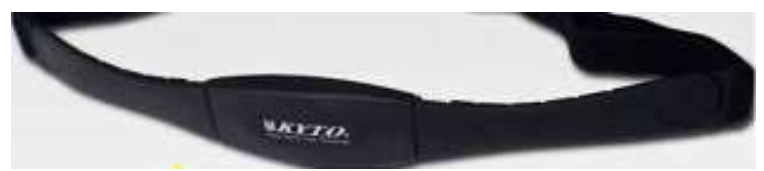

Figure 5. Heart rate monitor

\subsection{Neural Network Architecture}

In this section, neural network (Figure 6) is implemented to process generated input data for classifying the events in two classes: fall and not fall. The input data are: speed, aspect ratio, angle and heart rate. We generated 5000 such sets of values, each having correspondence with real life situations that can occur. We chose to have 2500 situations corresponding to non-fall situations and 2500 corresponding to fall situations. We decided that a fall situation occurs when we have measures of high speed, low aspect ratio, the angle under 45 degrees and a heart rate close to normal. For our network, we decided to implement a Multi-Layer Perceptron (MLP). The network is a feed forward network with Back Propagation. The output consists of 1 element that can be 1 or 0 . The value one has been assigned to the fall situation class and the value 0 correspond to the situation of non-fall. The training process allowed the neural network to automatically identify the regions in the input pattern space that contained the fall data points.

For all of the simulations we chose a sigmoid transfer function for the hidden layer and a linear transfer function for the output layer.

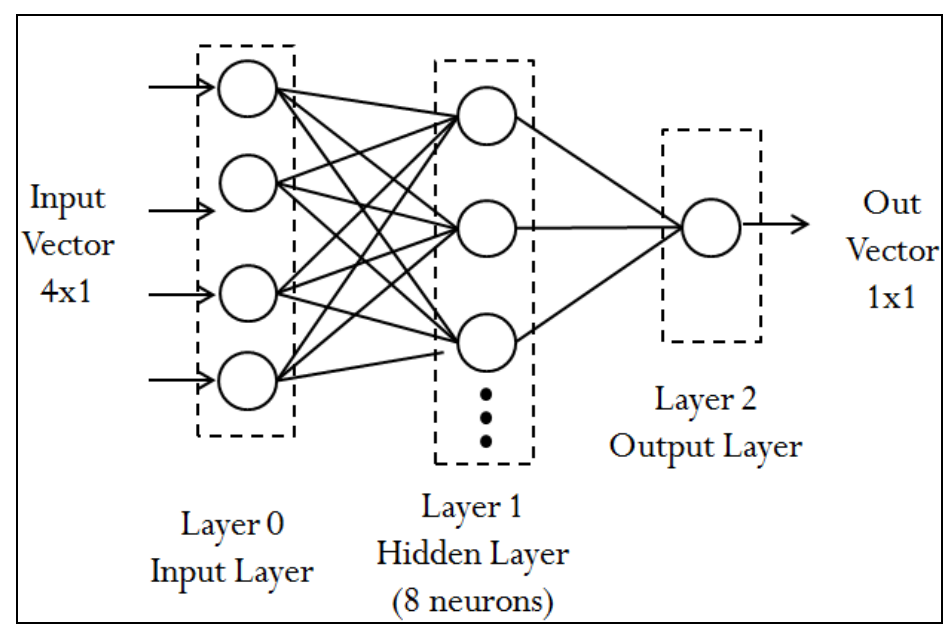

Figure 6. Neural network architecture

The network settings are presented below:

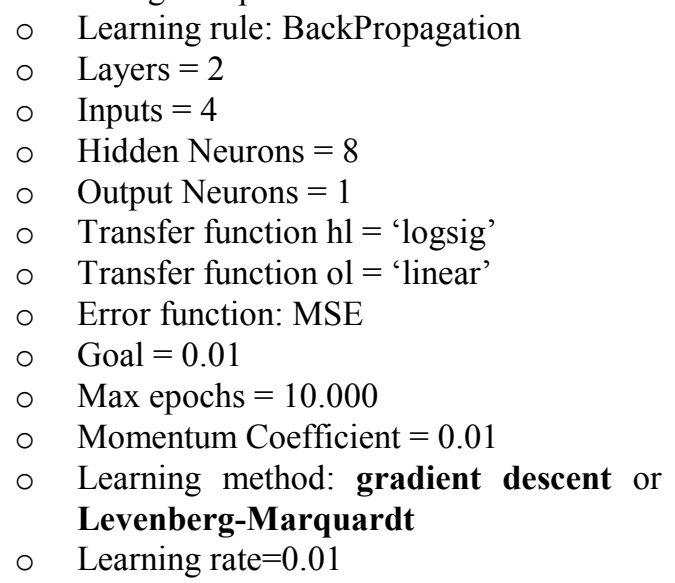

\section{Experimental Results and Discussion}

In our study, the video is considered as several captured images displayed successively. In order to evaluate the overall system performance, we apply the proposed MLP approach to the features extracted from the captured images and the heart rate accelerometer. In this section, we present the results obtained using the proposed surveillance system.

In principle, we can just use any raw input-output data to train our networks. However, in practice, it often helps the network to learn appropriately if we carry out some preprocessing of the training data before feeding it to the network. We should make sure that the training 
data is representative - it should not contain too many examples of one type at the expense of another. On the other hand, if one class of pattern is easy to learn, having large numbers of patterns from that class in the training set will only slow down the over-all learning process.

The ranges of our input are:

- $\quad$ Speed (pixel/s): from 90 to 700 ;

- Aspect ratio (height/width): from 0.15 to 6 ;

- $\quad$ Angle (degree): from 0 to 90;

- Heartbeat (bpm): from 70 to 200.

All inputs were normalized to $[-3 ; 3]$. The range of the output is $[0,1]$ (sigmoid function) so there is no need for scaling. The number of patterns that we used was 2500 for each class. Because in our approach we used batch training, there was no need for shuffling the order of the input patterns.

The quality of the system cannot be evaluated simply from a single test; instead it is necessary to carry out a statistical analysis on a series of tests.

There are 4 possible cases:

- True positives (TP): a fall occurs, the device detects it;

- $\quad$ False positives (FP): the device announces a fall, but it did not occur;

- True negative (TN): a normal (no fall) movement is performed, the device does not declare a fall;

- False negative (FN): a fall occurs but the device does not detect it;

We also define the following values:

- $\quad \mathrm{P}$ : the total number of falls;

- $\quad \mathrm{N}$ : the total number of non-falls.

Sensitivity is the capacity to detect a fall. It is given by the ratio between the number of detected falls and the total falls that occurred: Sensitivity $=\mathrm{TP} /(\mathrm{TP}+\mathrm{FN})$

Specificity is the capacity to avoid false positives. Intuitively it is the capacity to detect a fall only if it really occurs: Specificity $=\mathrm{TN} /(\mathrm{TN}+\mathrm{FP})$

Accuracy is the ability to distinguish and detect both fall (TP) and non-fall movement (TN):

Accuracy $=(\mathrm{TP}+\mathrm{TN}) /(\mathrm{P}+\mathrm{N})$

We tried to vary the number of hidden units from our network. We tried with 4, 8 and 12 hidden neurons and observed that the best performance was obtained for 4 neurons in the hidden neurons.

Table 1: Results for different learning methods

\begin{tabular}{|c|c|c|c|c|c|c|}
\hline \multirow{3}{*}{$\begin{array}{l}\text { Learning } \\
\text { method }\end{array}$} & \multirow{3}{*}{$\begin{array}{l}\text { Num } \\
\text { of } \\
\text { Epoc } \\
\text { hs }\end{array}$} & \multirow{3}{*}{ Goal } & \multicolumn{4}{|c|}{ Performance } \\
\hline & & & \multirow{2}{*}{$\begin{array}{l}\text { Training } \\
\text { lastepoch }\end{array}$} & \multicolumn{3}{|c|}{ Validation } \\
\hline & & & & $\begin{array}{l}\text { Sensi } \\
\text { tivity }\end{array}$ & $\begin{array}{l}\text { Speci } \\
\text { ficity }\end{array}$ & $\begin{array}{l}\text { Accu } \\
\text { racy }\end{array}$ \\
\hline traingdx & 56 & 0.1 & 0.0979 & 87.71 & 75.43 & 81.57 \\
\hline trainlm & 3 & 0.1 & 0.0358 & 99.53 & 93.17 & 96.59 \\
\hline traingdx & $\begin{array}{l}1000 \\
0\end{array}$ & 0.01 & 0.0156 & 92.37 & 96.12 & 98.07 \\
\hline trainlm & 7 & 0.01 & 0.00307 & 100 & 98.29 & 99.15 \\
\hline
\end{tabular}

Below, we present the results obtained using the proposed monitoring system.

Table 2: Fall detection results

\begin{tabular}{|l|l|l|l|l|}
\hline Speed & Ratio & Angle & $\begin{array}{l}\text { Heart } \\
\text { rate }\end{array}$ & Class \\
\hline 666 & 0.46 & 15 & 104 & Fall \\
\hline 689 & 0.99 & 5 & 70 & Fall \\
\hline 698 & 0.19 & 26 & 91 & Fall \\
\hline 650 & 0.56 & 16 & 86 & Fall \\
\hline 674 & 0.42 & 17 & 99 & Fall \\
\hline 583 & 4.4 & 60 & 110 & No Fall \\
\hline 328 & 1.12 & 20 & 90 & No Fall \\
\hline 147 & 0.32 & 45 & 86 & No Fall \\
\hline 423 & 2.03 & 50 & 77 & No Fall \\
\hline
\end{tabular}

\section{Conclusion}

Human fall is one of the major health problems for elderly people. Falls represent $38 \%$ of all home accidents and cause $70 \%$ of death in the $75+$ age group. Early detection of a fall is an important step in avoiding any serious injuries.

In an eldercare context, false alarms can be expensive. A false alarm may result in an alert being generated, such as a fall, requiring the intervention of a caregiver. Too many false alarms could result in a loss of trust, or 
worse, loss of use of the system. However, missing a single fall is the worst case scenario.

When developing such systems, we must ensure the privacy of the person, which can be satisfied here, as our systems are entirely automated and access to the images could be forbidden except in case of emergency. For instance, the system will send an alarm signal toward an outside resource (e.g. via Internet) if and only if an abnormal event is detected (e.g. falling).

Better performances and results can be obtained when different methods of acquiring data are combined (wearable devices + webcam images). Test results show that the system is reliable and reduce hospitalization costs by improving the living conditions of elderly people; also this work could be the first phase of implementing a multi-sensor network monitoring system for fall detection using more than one webcam also with other wearable devices (blood pressure, respiration...).

\section{References}

[1] N. Noury, T. Hervé, V. Rialle, G. Virone, E. Mercier. "Monitoring behavior in home using a smart fall sensor and position sensors". In IEEE-EMBS. Microtechnologies in Medicine \&Biology“, Lyon-France, Oct 2000; 607-610.

[2] N. Noury, A. Fleury, P. Rumeau, A.K. Bourke, G. O. Laighin, V. Rialle, J.E. Lundy, "Fall Detection - Principles and Methods," 29th Annual Int. Conf. of the IEEE Engineering in Medicine and Biology Society, Lion (France), pp. 1663-1666, August 2007.

[3] G. Williams et al. "A smart fall and activity monitor for telecare application ". Int. Conf. IEEEEMBS, Hong-Kong, 1998, pp.1151-1154Conference on, 13(16):493 - 498, 2008.

[4] Yamaguchi. "Monitoring behavior in home using positioning sensors" Int. Conf. IEEE-EMBS, Hong-Kong, 1998; 1977-79

[5] Report on seniors's falls in canada. Public Health Agency of Canada, Division of Aging and Seniors, 2005.

\section{[6] http://www.lifelinesys.com}

[7] B. T"oreyin, Y. Dedeoglu, and A. C, etin. HMM based Falling person detection using both audio and video. In IEEE International Workshop on Human-Computer Interaction, Beijing, China, 2005

[8] S.-G.Miaou, P.-H. Sung, and C.-Y. Huang, "A Customized Human Fall Detection System Using OmniCamera Images and Personal Information" Proc of Distributed Diagnosis and Home Healthcare(D2H2) Conference, 2006
[9] R. Cucchiara, A.Pratti, and R.Vezani, "An Intelligent Surveillance System for Dangerous Situation Detection in home Environments", in Intelligenza artificable, vol.1, n.1, pp. 11-15, 2004.

[10] Nait CH, McKenna SJ. "Activity summarisation and fall detection in a supportive home environment", Int. Conf. on Pattern Recognition (ICPR), 2004

[11] Tao, J., M.Turjo, M.-F. Wong, M.Wang, "Fall Incidents Detection for Intelligent Video Surveillance", ICICS2005

[12] C.Rougier, J.Meunier, A. St-Arnaud, J.Rousseau, "Monocular 3D Head Tracking to Detect Falls of Elderly People", International Conference of IEEE Engineering in Medicine and Biology Society, Sept 2006

[13] Arie Hans Nasution and S. Emmanuel; Intelligent Video Surveillance for Monitoring Elderly in Home Environments, International Workshop on Multimedia Signal Processing (MMSP), Greece, October 2007

[14] Anh Dinh \&Tao Wang, 'Bandage-Size Non-ECG Heart Rate Monitor Using ZigBee Wireless Link',

International Conference On Bioinformatics and Biomedical Technology (ICBBT) ,Page 160-163, 2010.

[15] Yang Tang, Guitao Cao, Hao Li, "The design of electronic heart sound stethoscope based on Bluetooth" 4th International Conference On Bioinformatics and Biomedical Engineering (ICBBE),

Page No. 1-4, 2010.

[16] Jia-Ren Chang Chien, Cheng-Chi Tsi, "The Implementation of a Bluetooth-Based Wireless Phonocardio-Diagnosis System", International Conference on Networking, Sensing and Control,

March 2004. 